\title{
Synthesis of 2-(2-fluorophenyl)-2-methylamino-cyclohexanone as a new ketamine derivative
}

\author{
Abolghasem Moghimi, ${ }^{a}$ Siyavash Rahmani, ${ }^{\mathrm{a}, \mathrm{b},{ }^{*}}$ Reza Zare, ${ }^{\mathrm{a}}$ Morteza Sadeghzadeh ${ }^{\mathrm{a}}$ \\ ${ }^{a}$ Department of Chemistry, Imam Hossein University, P.O. Box 16575-347, Tehran, Iran. \\ ${ }^{\mathrm{b}}$ Department of Nuclear Medicine, The Educational, Research and Clinical Center, Dr. Masih Daneshvari Hospital, Tehran, Iran.
}

\section{Supplementary Material}

Table of Contents

Page

1. Spectral data $2-4$

2. Copies of ${ }^{1} \mathrm{H}$ and ${ }^{13} \mathrm{C}$ NMR spectra $\quad 5-16$

$\begin{array}{ll}\text { 3. Copies of mass spectra for } 7 & 17\end{array}$

\footnotetext{
* Corresponding Author:

E-mail: Siyavash.Rahmani@yahoo.com
} 


\section{Spectral data}

\subsection{Cyclopentyl-(2-fluorophenyl)-ketone (2).}

Yield: (90\%); light yellow oil. IR (KBr): 2955, 2869, 1679, 1608, 1479, 1451, 1271, 1211, 756 $\mathrm{cm}^{-1} ;{ }^{1} \mathrm{H}$ NMR $\left(250 \mathrm{MHz}, \mathrm{CDCl}_{3}\right) \delta: 1.6-1.72(4 \mathrm{H}, \mathrm{m}), 1.86-1.93(4 \mathrm{H}, \mathrm{m}), 3.59-3.66(1 \mathrm{H}, \mathrm{m})$, $7.06-7.23(2 \mathrm{H}, \mathrm{m}), 7.42-7.51(1 \mathrm{H}, \mathrm{m}), 7.74-7.81(1 \mathrm{H}, \mathrm{m}) ;{ }^{13} \mathrm{C} \mathrm{NMR}\left(62.90 \mathrm{MHz}, \mathrm{CDCl}_{3}\right) \delta$ : $26.09\left(2 \mathrm{CH}_{2}, \mathrm{~s}\right), 29.27\left(2 \mathrm{CH}_{2}, \mathrm{~d}, J=0.64 \mathrm{~Hz}\right), 50.98(\mathrm{CH}, \mathrm{d}, J=5.77 \mathrm{~Hz}), 116.42(\mathrm{CH}, \mathrm{d}, J=23.9$ $\mathrm{Hz}), 124.39(\mathrm{CH}, \mathrm{d}, J=3.45 \mathrm{~Hz}), 126.21$ (quat-C, d, $J=13.02 \mathrm{~Hz}), 130.83(\mathrm{CH}, \mathrm{d}, J=2.89 \mathrm{~Hz}$ ), 133.92(CH, d, $J=8.99 \mathrm{~Hz}), 159.40\left(\right.$ quat-C, d, $\left.J_{C-F}=253.74 \mathrm{~Hz}\right), 201.51($ quat-C, C=O) ppm.

\section{$1.2 \alpha$-bromocyclopentyl-(2-fluorophenyl)-ketone (3)}

Yield: (96\%); brown oil. ${ }^{1} \mathrm{H}$ NMR $\left(300 \mathrm{MHz}, \mathrm{CDCl}_{3}\right) \delta 1.83(2 \mathrm{H}, \mathrm{m}), 2.02(2 \mathrm{H}, \mathrm{m}), 2.37(4 \mathrm{H}, \mathrm{m})$, 7.08- 7.21(2H, m), $7.41-7.48(1 \mathrm{H}, \mathrm{m}), 7.74-7.78(1 \mathrm{H}, \mathrm{m}) ;{ }^{13} \mathrm{C} \mathrm{NMR}\left(75 \mathrm{MHz}, \mathrm{CDCl}_{3}\right) \delta$ 23.22( $\left.\mathrm{CH}_{2}, \mathrm{~s}\right), 40.22\left(\mathrm{CH}_{2}, \mathrm{~s}\right), 73.81($ quat-C, s), $116(\mathrm{CH}, \mathrm{d}, J=22 \mathrm{~Hz}), 123(\mathrm{CH}, \mathrm{d}, J=3.22 \mathrm{~Hz})$, 126(quat-C, d, $J=14.8 \mathrm{~Hz}), 130(\mathrm{CH}, \mathrm{d}, J=2.3 \mathrm{~Hz}), 132(\mathrm{CH}, \mathrm{d}, J=8.47 \mathrm{~Hz}), 159$ (quat-C, d, $J_{C-F}$ $=250.8 \mathrm{~Hz}$ ), 196.37(quat-C, $\mathrm{C}=\mathrm{O}$ ).

\section{$1.3 \alpha$-hydroxycyclopentyl-(2-flourophenyl)-N-methylamine (4)}

Yield: (81\%); shiny crystals. Mp $41.5-43.5^{\circ} \mathrm{C}$; IR (KBr): 3297, 2971, 2873, 1656, 996, $765 \mathrm{~cm}^{-}$

${ }^{1} ;{ }^{1} \mathrm{H}$ NMR $\left(300 \mathrm{MHz}, \mathrm{CDCl}_{3}\right) \delta: 1.26-1.69(5 \mathrm{H}, \mathrm{m}), 1.91-2.2(3 \mathrm{H}, \mathrm{m}), 3.03(3 \mathrm{H}, \mathrm{s}), 5.48(1 \mathrm{H})$, $7.03-7.27(3 \mathrm{H}, \mathrm{m}), 7.39-7.51(1 \mathrm{H}, \mathrm{m}) ;{ }^{13} \mathrm{C} \mathrm{NMR}\left(75 \mathrm{MHz}, \mathrm{CDCl}_{3}\right) \delta: 23.53\left(\mathrm{CH}_{2}\right), 23.84\left(\mathrm{CH}_{2}\right)$, 
37.8 $\left(\mathrm{CH}_{2}\right), 38.01\left(\mathrm{CH}_{2}\right), 39.68\left(\mathrm{CH}_{3}\right), 84.08($ quat-C), $115.7(\mathrm{CH}, \mathrm{d}, J=24.90 \mathrm{~Hz}), 122.16($ quat-C, d, $J=19.62 \mathrm{~Hz}), 124.18(\mathrm{CH}, \mathrm{d}, J=2.41 \mathrm{~Hz}), 129.12(\mathrm{CH}, \mathrm{d}, J=4.52 \mathrm{~Hz}), 130.62(\mathrm{CH}, \mathrm{d}, J=$ 7.54 Hz), 156.61(quat-C, d, $J_{C-F}=246.425 \mathrm{~Hz}$ ), 171.12(quat-C, C=N) ppm.

\subsection{2-(2-fluorophenyl)-2-methylamino-cyclohexanone (5)}

Yield: (53\%); brown oil. IR (KBr): 3371, 2942, 2863, 1714, 1485, $759 \mathrm{~cm}^{-1} ;{ }^{1} \mathrm{H}$ NMR (300MHz, $\left.\mathrm{CDCl}_{3}\right) \delta: 1.70-1.80(4 \mathrm{H}, \mathrm{m}), 1.93(1 \mathrm{H}, \mathrm{s}), 2.11(3 \mathrm{H}, \mathrm{s}), 2.30-2.47(2 \mathrm{H}, \mathrm{m}), 2.55(1 \mathrm{H}, \mathrm{s}), 2.75-$ 2.84(1H, m), 7.04(1H, m), 7.17 - 7.22(1H, m), 7.29(1H, m), 7.43(1H, m); ${ }^{13} \mathrm{C}$ NMR $(75 \mathrm{MHz}$ $\left.\mathrm{CDCl}_{3}\right) \delta: \quad 21.95\left(\mathrm{CH}_{2}\right), \quad 28.48\left(\mathrm{CH}_{2}\right), 29.20\left(\mathrm{CH}_{3}\right), \quad 38.18\left(\mathrm{CH}_{2}\right), \quad 39.38\left(\mathrm{CH}_{2}\right), \quad 68.33$ (quat-C), $116.13(1 \mathrm{CH}, \mathrm{d}, J=23.39 \mathrm{~Hz}), 124.24(\mathrm{CH}, \mathrm{d}, J=3.01 \mathrm{~Hz}), 126.73$ (quat-C, d, $J=12.83 \mathrm{~Hz}$ ), $128.83(\mathrm{CH}, \mathrm{d}, J=4.52 \mathrm{~Hz}), 129.47(\mathrm{CH}, \mathrm{d}, J=8.30 \mathrm{~Hz}), 161\left(\right.$ quat-C, d, $\left.J_{C-F}=246.80 \mathrm{~Hz}\right)$, $210($ quat-C, $\mathrm{C}=\mathrm{O}) \mathrm{ppm}$.

\section{5 (2-fluorophenyl)(1-hydroxycyclopentyl)methanone (6)}

Brown oil. IR (KBr): 3480, 2957, 1687, 1611, 1451, 1279, 1219, $753 \mathrm{~cm}^{-1} ;{ }^{1} \mathrm{H}$ NMR (300MHz, $\left.\mathrm{CDCl}_{3}\right) \delta: 1.79-1.92(6 \mathrm{H}, \mathrm{m}), 2.18-2.22(2 \mathrm{H}, \mathrm{m}), 2.90(1 \mathrm{H}), 7.13-7.51(4 \mathrm{H}, \mathrm{m}) ;{ }^{13} \mathrm{C}$ NMR $(75$ $\left.\mathrm{MHz}, \mathrm{CDCl}_{3}\right) \delta: 24.75\left(2 \mathrm{CH}_{2}\right), 39.37\left(\mathrm{CH}_{2}\right), 39.40\left(\mathrm{CH}_{2}\right), 88.30$ (quat-C), $116.07(\mathrm{CH}, \mathrm{d}, J=$ $22.5 \mathrm{~Hz}), 124(\mathrm{CH}, \mathrm{d}, J=3.22 \mathrm{~Hz}), 126($ quat-C, d, $J=16.5 \mathrm{~Hz}), 129(\mathrm{CH}, \mathrm{d}, J=3.5 \mathrm{~Hz}), 132(\mathrm{CH}$, d, $J=8.4 \mathrm{~Hz}$ ), 159(quat-C, d, $J_{C-F}=247.42 \mathrm{~Hz}$ ), 206(quat-C, C=O) ppm. 


\subsection{1-(2-fluorophenyl)-N-methyl-2-oxocyclohexanaminium chloride (7)}

Mp $258-260{ }^{\circ} \mathrm{C}$.; IR (KBr): 3439, 2975, 2929, 2869, 2663, 1725, 1556, 1491, 1451, 1231, 770 $\mathrm{cm}^{-1} .{ }^{1} \mathrm{H}$ NMR $\left(300 \mathrm{MHz}, \mathrm{D}_{2} \mathrm{O}\right) \delta: 1.58(2 \mathrm{H}, \mathrm{m}), 1.76-1.79(2 \mathrm{H}, \mathrm{m}), 1.94(1 \mathrm{H}, \mathrm{m}), 2.27(3 \mathrm{H}, \mathrm{s})$, $2.387-2.45(2 \mathrm{H}, \mathrm{m}), 3.11(1 \mathrm{H}, \mathrm{d}, J=13.8 \mathrm{~Hz}), 7.13-7.20(1 \mathrm{H}, \mathrm{m}), 7.32-7.34(1 \mathrm{H}, \mathrm{m})$, 7.49(1H, m), $7.62-7.64(1 \mathrm{H}, \mathrm{m}) . \dot{ }^{13} \mathrm{C}$ NMR $\left(75 \mathrm{MHz}, \mathrm{D}_{2} \mathrm{O}\right) \delta: 20.95\left(\mathrm{CH}_{2}\right), 26.38\left(\mathrm{CH}_{2}\right)$, 28.67( $\left(\mathrm{CH}_{3}\right), 33.93\left(\mathrm{CH}_{2}\right), 38.46\left(\mathrm{CH}_{2}\right), 69.43$ (quat-C), $116.9(\mathrm{CH}), 117.12$ (quat-C, d, $J=6.792$ $\mathrm{Hz}), 125.73(\mathrm{CH}, \mathrm{d}, J=2.26 \mathrm{~Hz}), 130.15(\mathrm{CH}), 133.53(\mathrm{CH}, \mathrm{d}, J=9.057 \mathrm{~Hz}), 160.8$ (quat-C, d, $J=$ $246.8 \mathrm{~Hz}$ ), 209(quat- $\mathrm{C}, \mathrm{C}=\mathrm{O}) \mathrm{ppm} . \mathrm{MS} \mathrm{m} / \mathrm{z}$ (percent abundance): $221.2\left[\mathrm{M}^{+}\right](10 \%), 193(45 \%)$, 165 (30\%), 164 (70\%), 109(25\%). Anal. calcd. For $\mathrm{C}_{13} \mathrm{H}_{16} \mathrm{FNO} . \mathrm{HCl}: \mathrm{C}, 60.60 ; \mathrm{H}, 6.60 ; \mathrm{N}$, 5.43\%. Found C, 60.1; H, 6.58; N, 5.41\%. 
2. Copies of ${ }^{1} \mathrm{H}$ and ${ }^{13} \mathrm{C}$ NMR spectra

Copy of ${ }^{1} \mathrm{H}-\mathrm{NMR}\left(\mathrm{CDCl}_{3}\right)$ spectra for 2

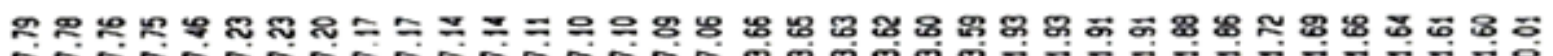

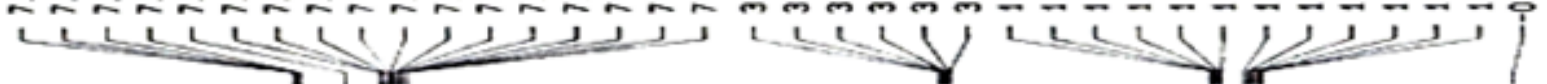

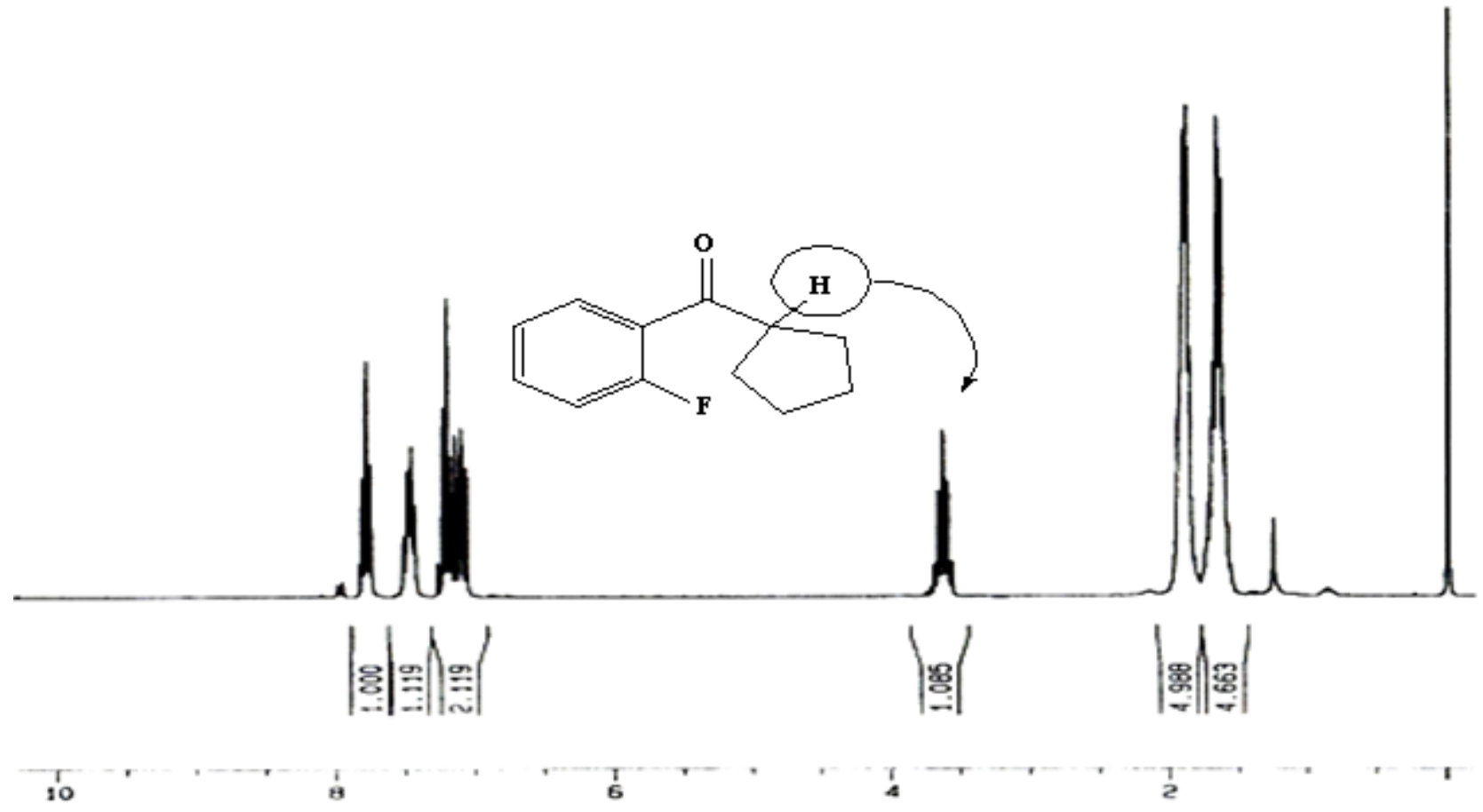




\section{Copy of ${ }^{13} \mathrm{C}$-NMR $\left(\mathrm{CDCl}_{3}\right)$ spectra for 2}

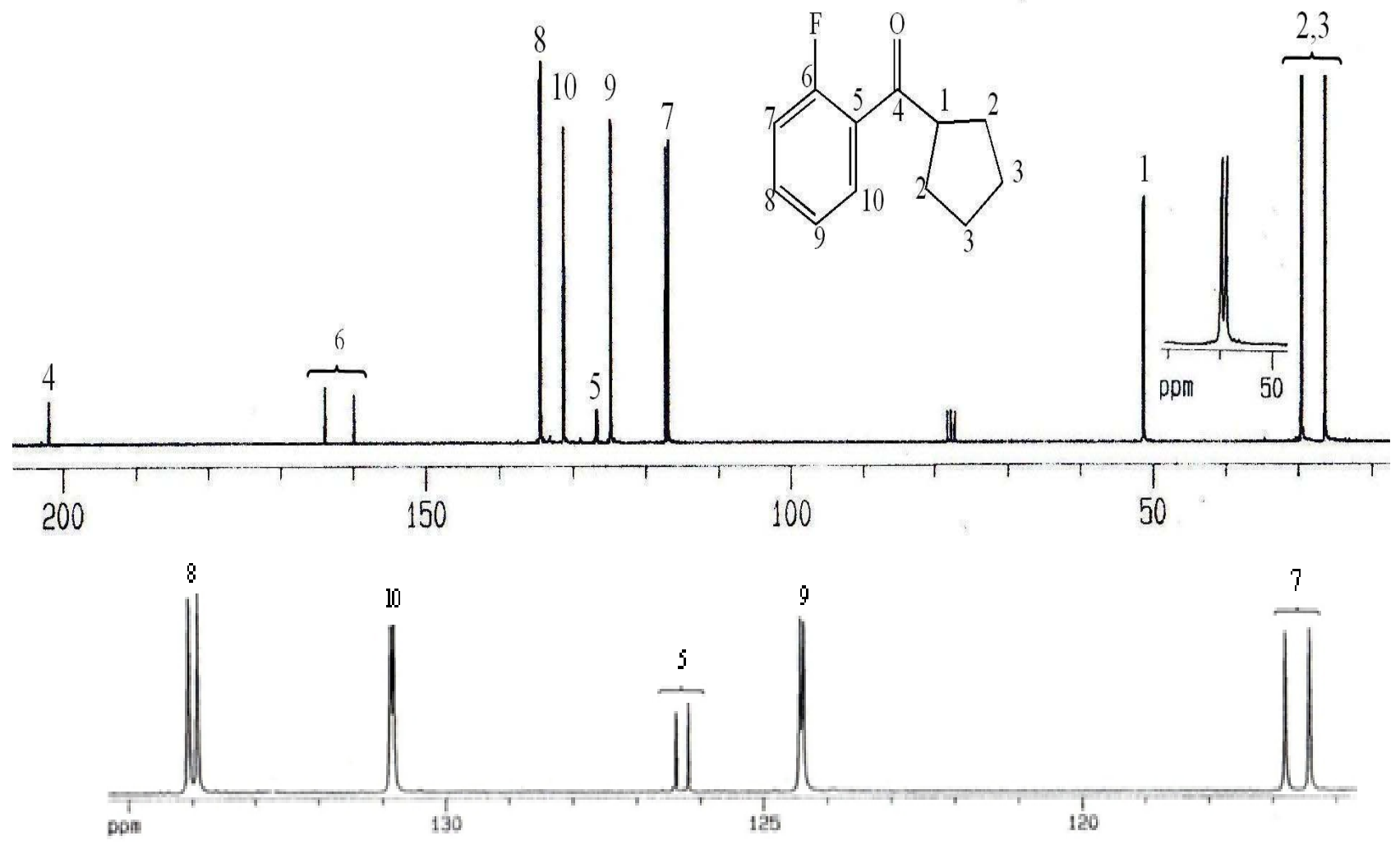




\section{Copy of ${ }^{1} \mathrm{H}$-NMR $\left(\mathrm{CDCl}_{3}\right)$ spectra for 3}

究

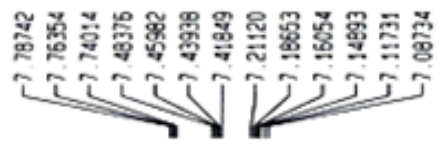

余总岕

$\pi \tilde{V}$

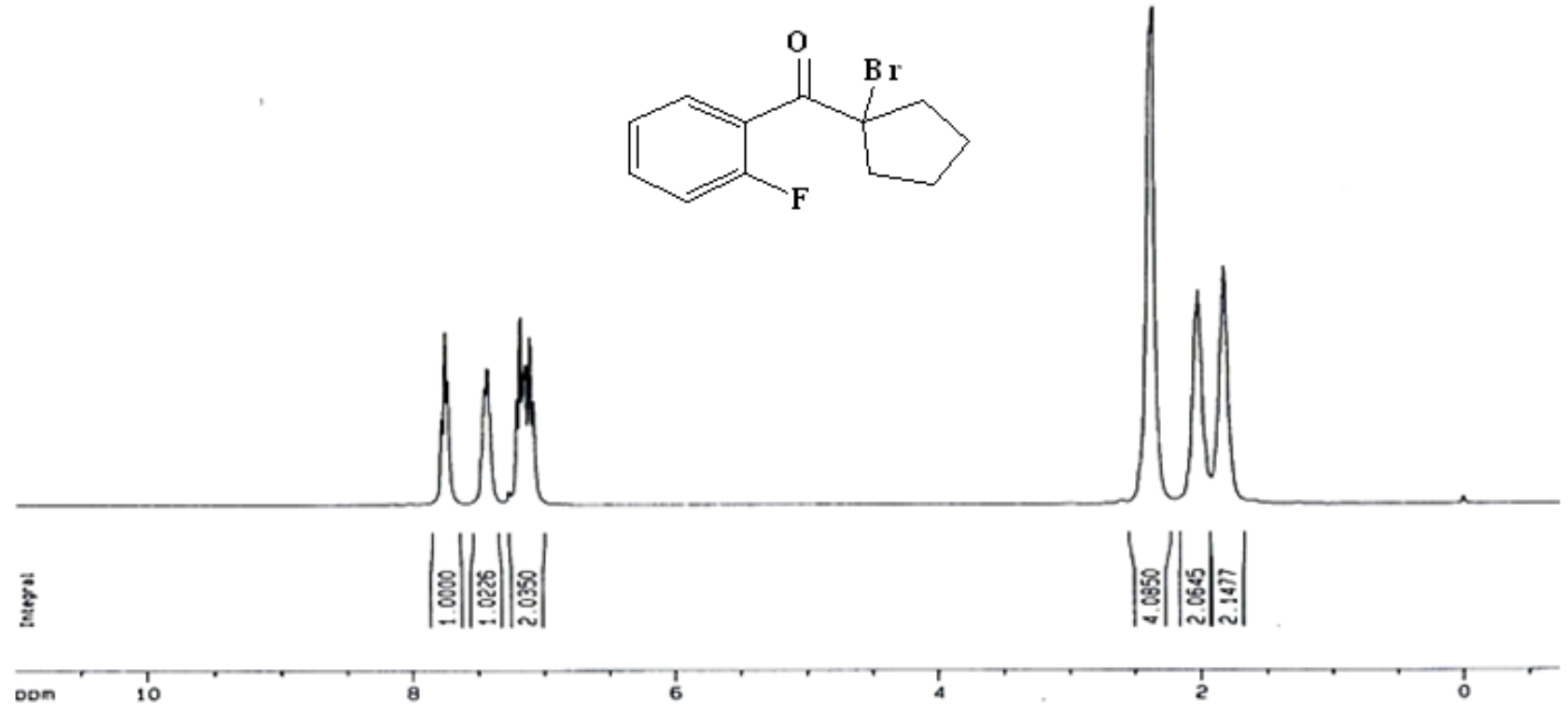




\section{Copy of ${ }^{13} \mathrm{C}$-NMR $\left(\mathrm{CDCl}_{3}\right)$ spectra for 3}
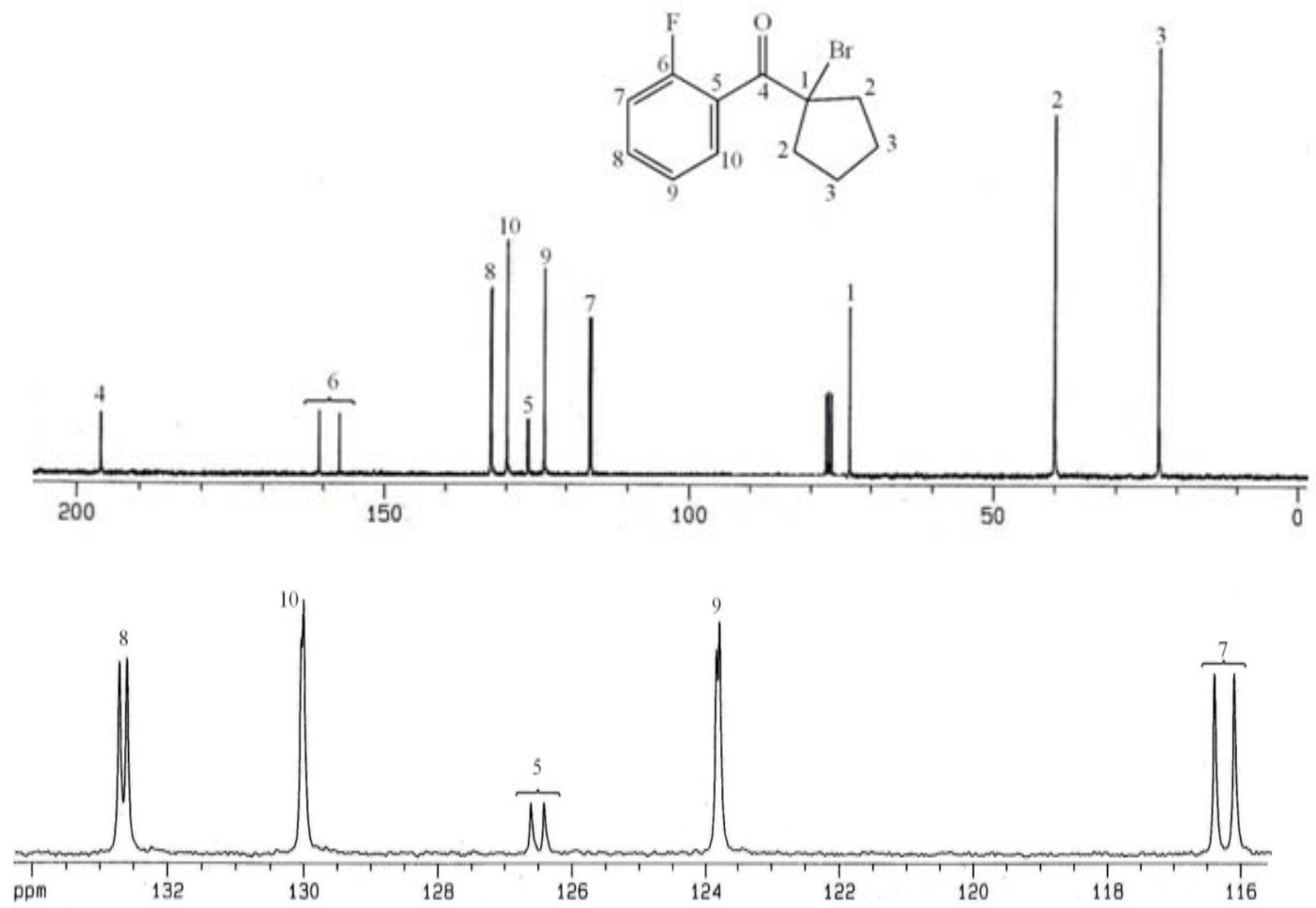


\section{Copy of ${ }^{1} \mathrm{H}$-NMR $\left(\mathrm{CDCl}_{3}\right)$ spectra for 4}

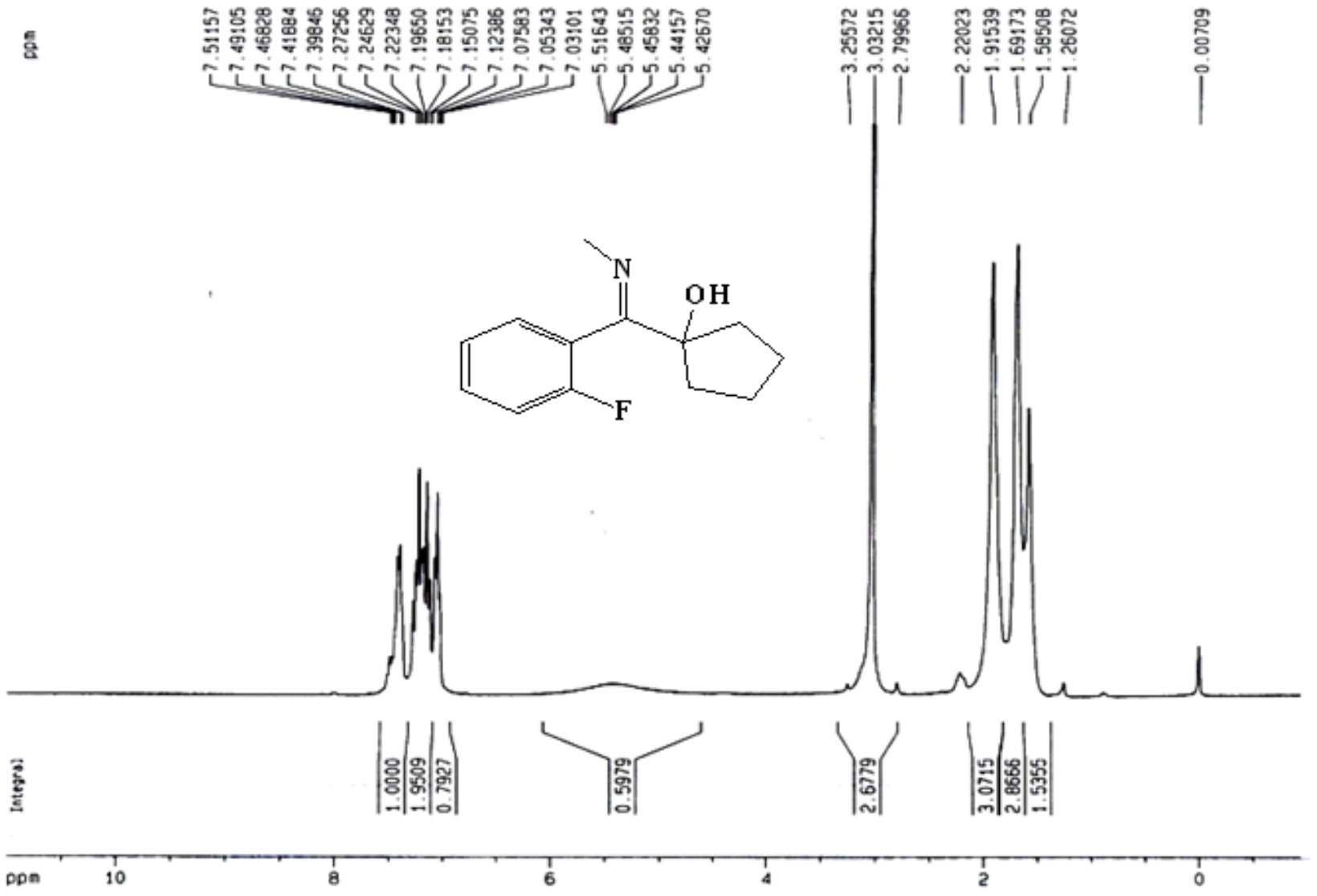


Copy of ${ }^{13} \mathrm{C}$-NMR $\left(\mathrm{CDCl}_{3}\right)$ spectra for 4
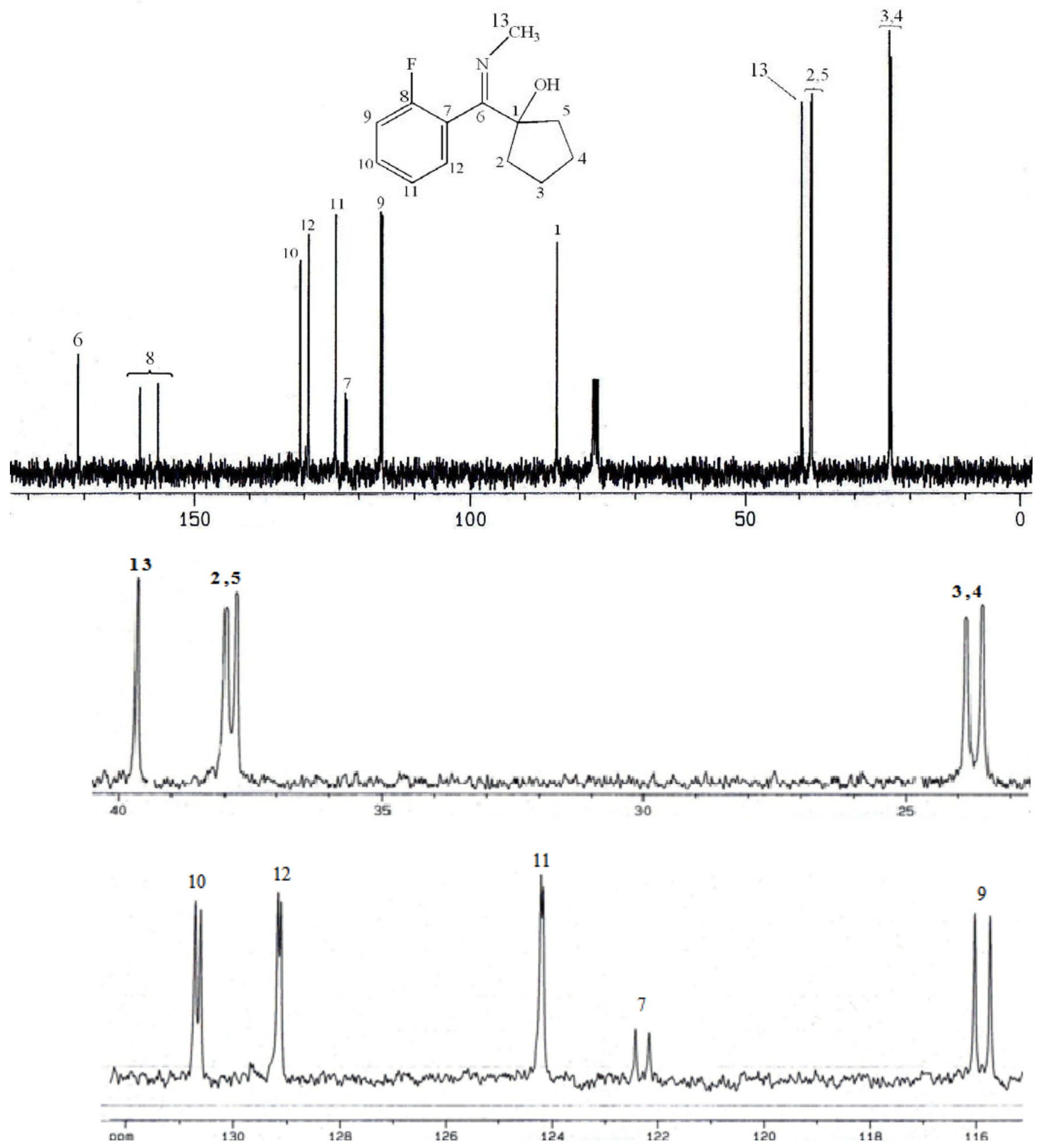


\section{Copy of ${ }^{1} \mathrm{H}$-NMR $\left(\mathrm{CDCl}_{3}\right)$ spectra for 5}

ธิ

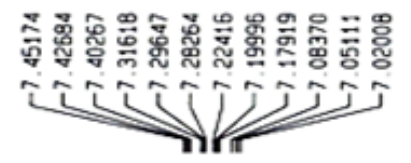

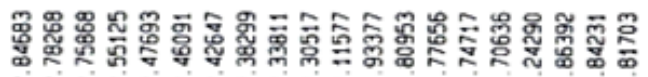
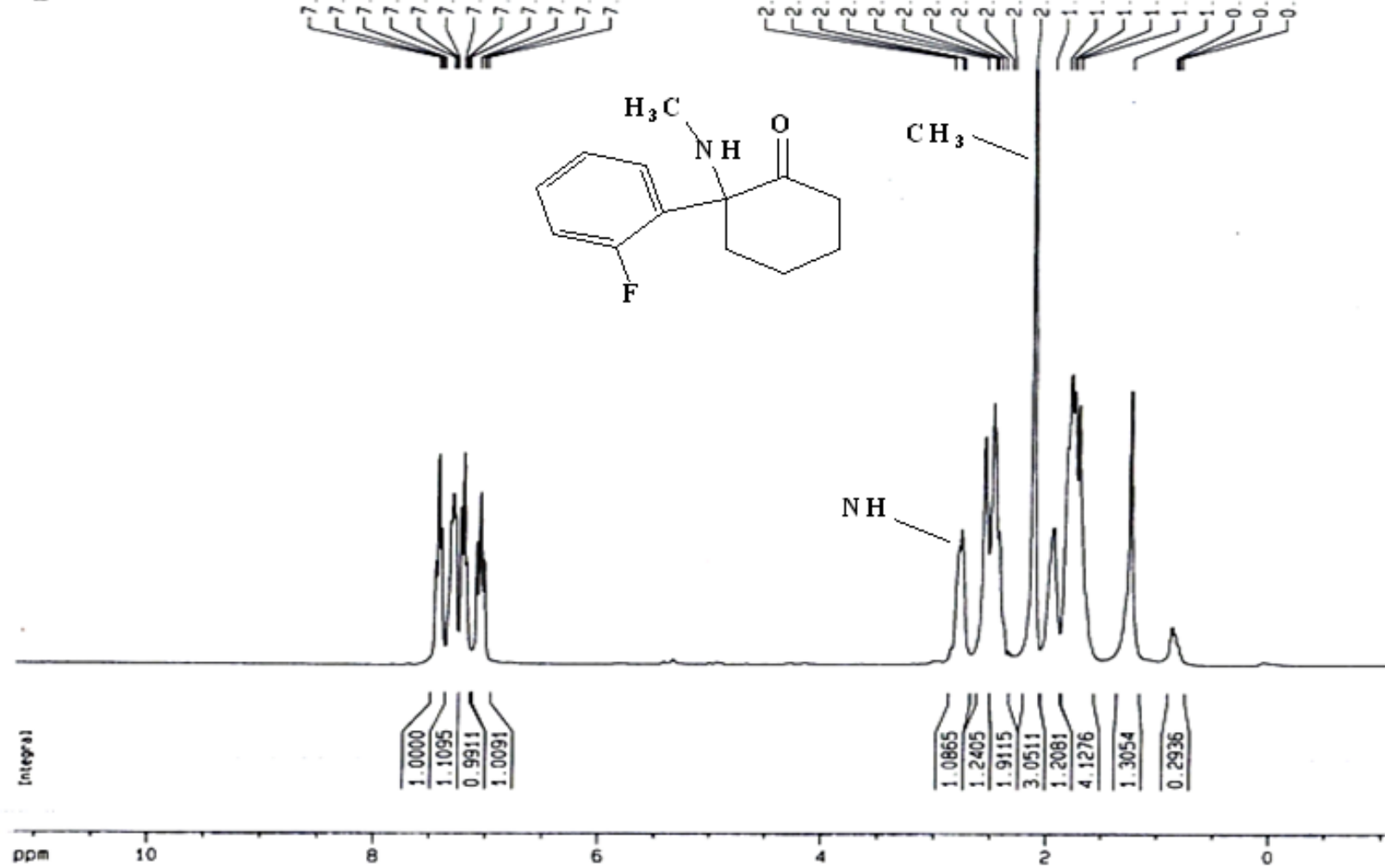

71 1 II

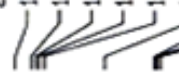


Copy of ${ }^{13} \mathrm{C}$-NMR $\left(\mathrm{CDCl}_{3}\right)$ spectra for 5

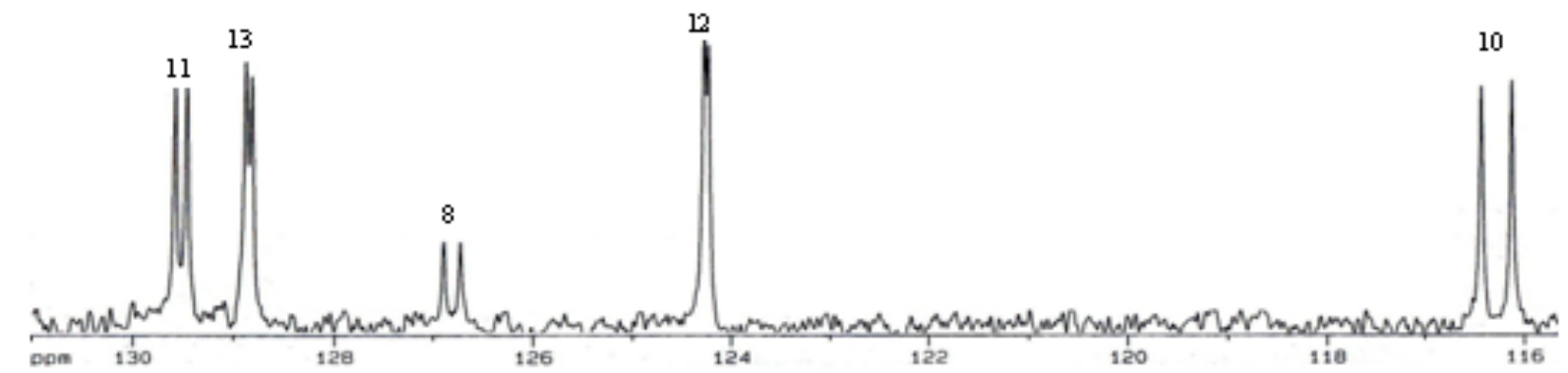<smiles>CCC1(c2ccccc2F)CCCCC1=O</smiles>

(5)
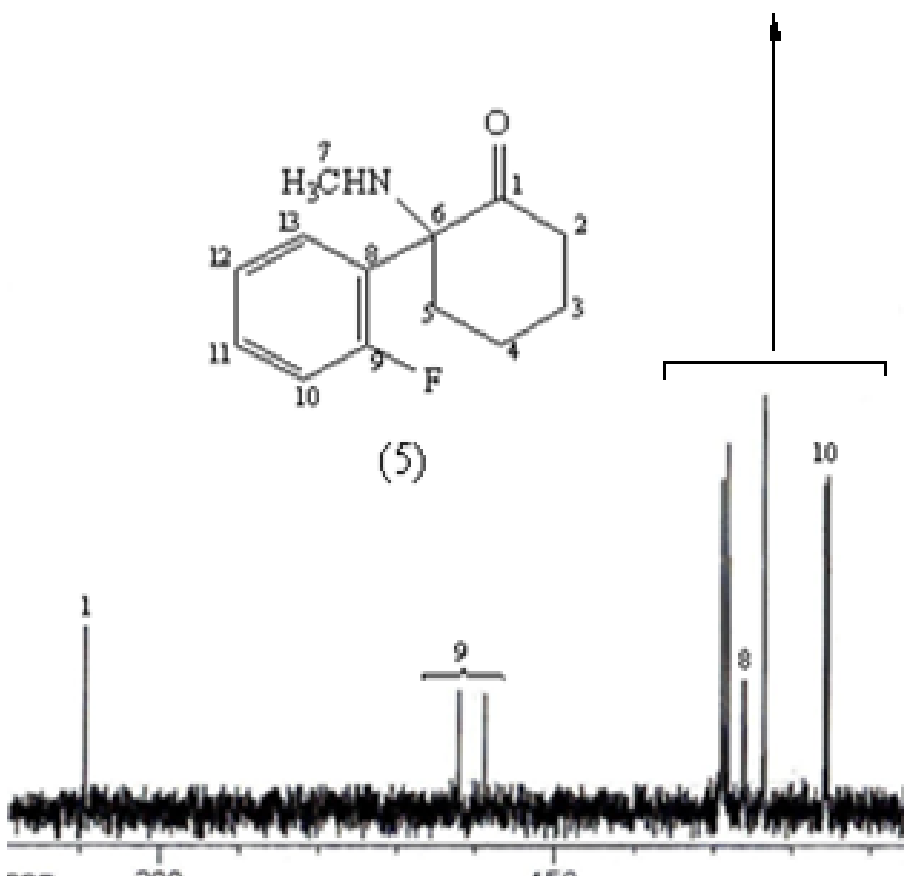

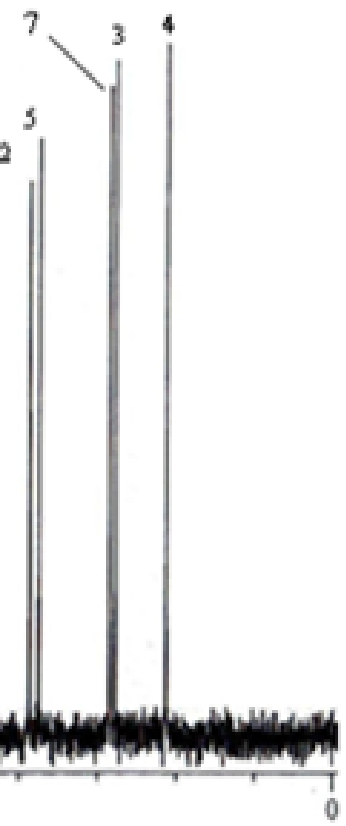




\section{Copy of ${ }^{1} \mathrm{H}$-NMR $\left(\mathrm{CDCl}_{3}\right)$ spectra for 6}

言

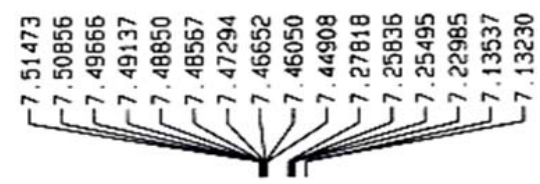

윰
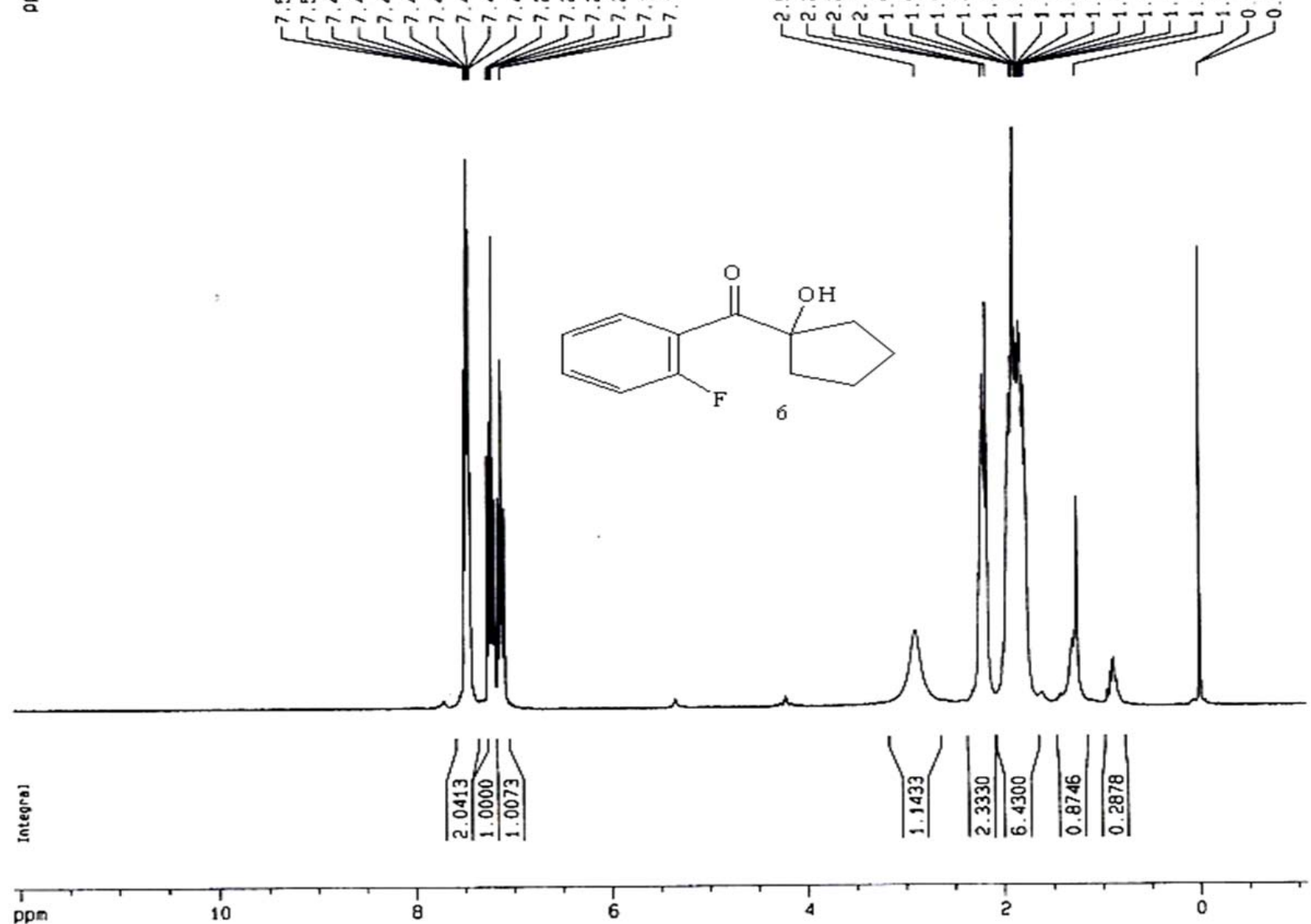
Copy of ${ }^{13} \mathrm{C}$-NMR $\left(\mathrm{CDCl}_{3}\right)$ spectra for 6
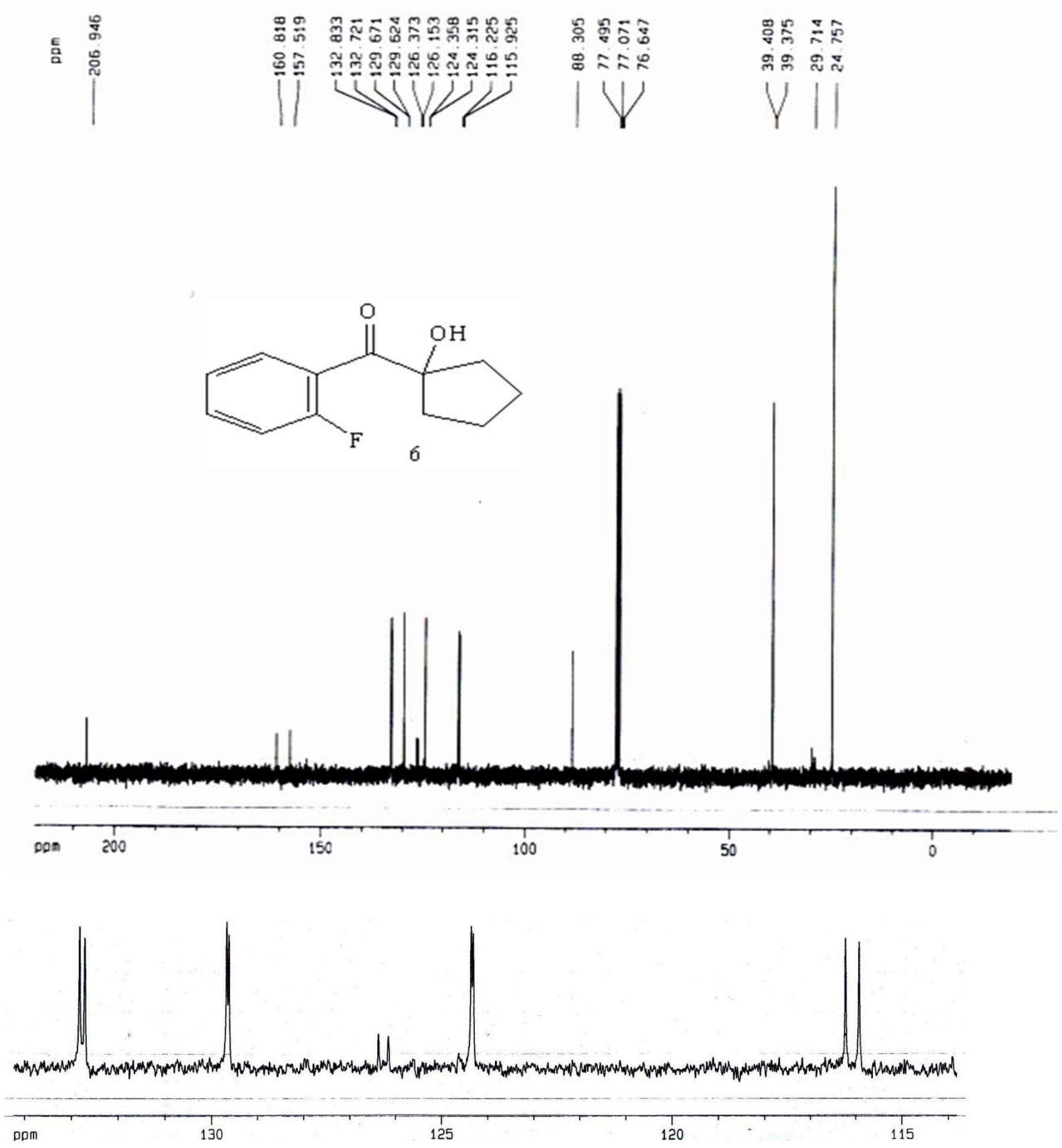


\section{Copy of ${ }^{1} \mathrm{H}$-NMR $\left(\mathrm{D}_{2} \mathrm{O}\right)$ spectra for 7}

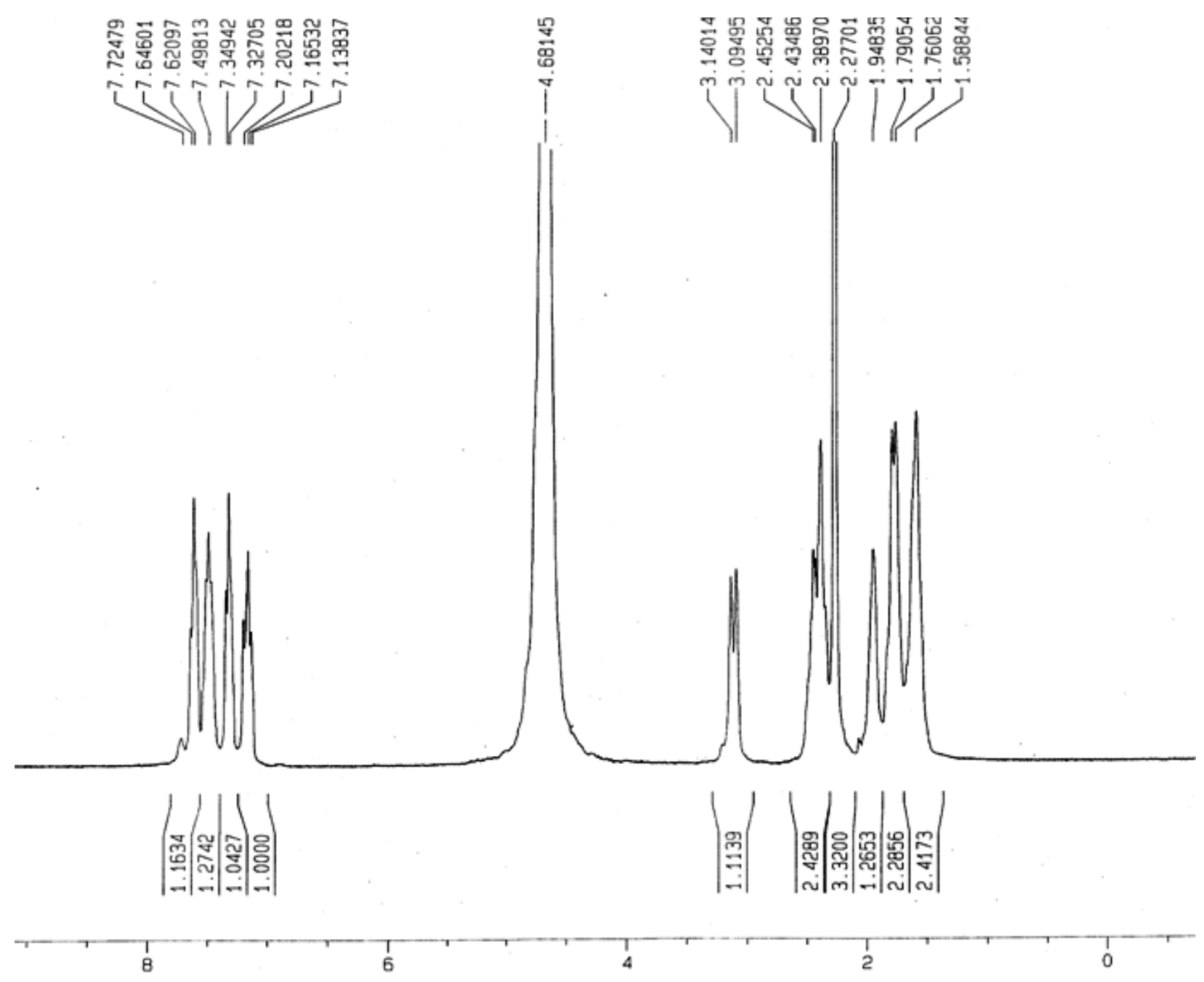


Copy of ${ }^{13} \mathrm{C}$-NMR $\left(\mathrm{D}_{2} \mathrm{O}\right)$ spectra for 7
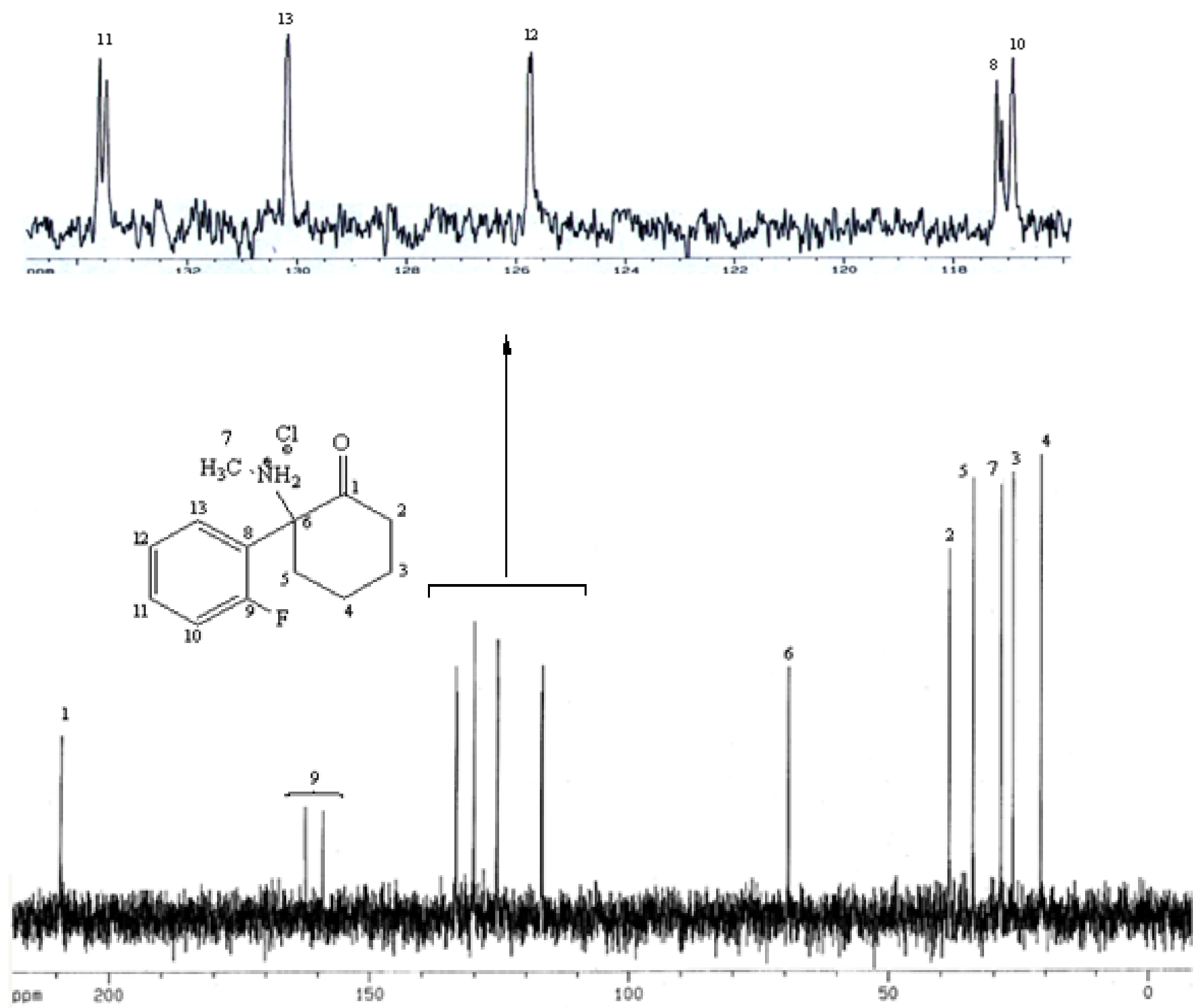


\section{Copy of mass spectra for 7}

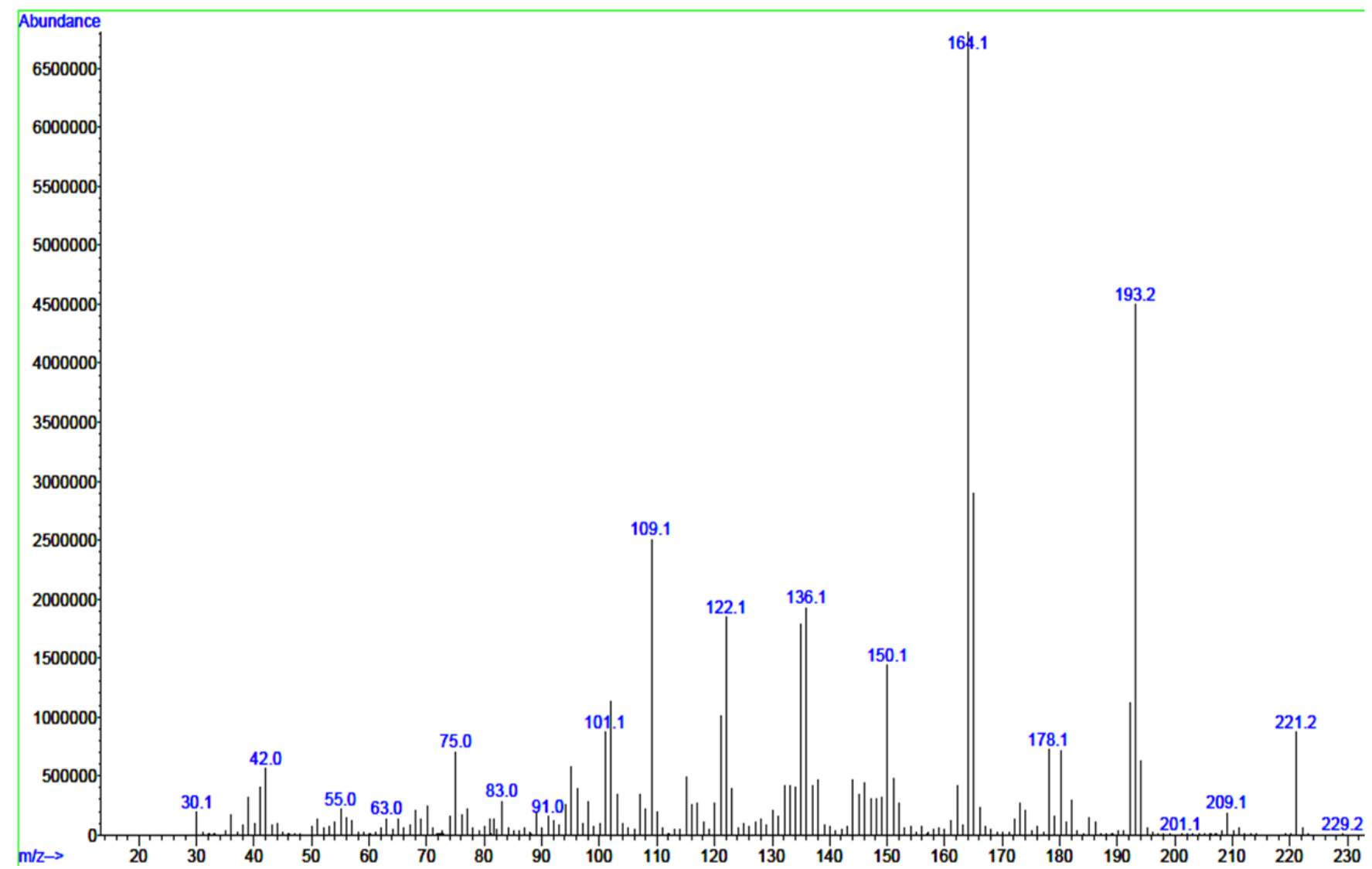

